\title{
Reversible Light-Induced Solubility of Disperse Red 1 Dye in a Hydroxypropyl cellulose Matrix
}

\section{Agnieszka Pawlicka ${ }^{1, *}$, Rodrigo C.Sabadini ${ }^{1}$, and Jean-Michel Nunzi ${ }^{2,3}$}

1. IQSC-Universidade de São Paulo, Av. Trabalhador Sãocarlense 400, 13566-590 São Carlos-SP, Brazil

2. Queens University, Dept. Phys. \& Engn. Phys. \& Astron. and Dept. Chem., Kingston, ON K7L 3N6, Canada

3. Aix Marseille Univ, Université de Toulon, CNRS, IM2NP UMR 7334, Yncrea Méditerranée, ISEN-Toulon, Maison du Numérique et de l'Innovation, Place G. Pompidou, 83000 Toulon, France

*e-mail:agnieszka@iqsc.usp.br

\begin{abstract}
We present results on reversible light-induced solubility of Disperse Red 1 (DR1) dye in a hydroxypropyl cellulose (HPC) matrix. The samples were prepared by dissolution of both DR1 and HPC in acetone and left to dry overnight in air to form uniform coatings. Visible small darker spots attributed to DR1 aggregates formed spontaneously. It is observed that both heating and illumination of the samples with visible light promote the appearance of a dark red coloration inside the heated or illuminated regions. This new phenomenon is attributed to a dissolution of dye and/or disruption of DR1 aggregates. It is confirmed by visible light and FTIR spectroscopies. UV-Vis spectra evidence disappearance of a band at $400 \mathrm{~nm}$, which is attributed to DR1 aggregates, upon illumination of the samples with visible light. FTIR spectra show new bands at 1600 , 1508 , and $1342 \mathrm{~cm}^{-1}$ that were assigned to symmetric and asymmetric stretching modes of $\mathrm{NO}_{2}$ of the dissolved DR1. Results evidence a dye solubilization process throughout repeated trans-cis isomery induced by visible light. This process is also a new feature of the light induced molecular movements of azo-dyes and may be used in future for the photo-command of polymer properties and/or light storage. This work opens new
\end{abstract}


perspectives to use natural polymers and composites in photonic applications. It also reveals the strong potential of azo-materials as light powered molecular motors.

\section{Keywords}

Natural polymer derivative; azo-dye; photoactive material; light triggered materials properties; hydrogen bonding.

\section{Introduction}

Azo chromophores have been extensively studied for their photo-active properties, which are related to the trans-cis isomery under illumination with visible light (Cojocariu and Rochon 2004). They found applications into holography (Zilker et al. 1998), nonlinearoptics (Apostoluk et al. 2002), surface patterning (Viswanathan et al. 1999), and photoactivated devices (Sentein et al. 1997). Furthermore, these can be used for technological developments into light extracting or coupling structures for plastic optoelectronics like organic light emitting diodes (Hubert et al. 2004) and polymer solar cells (Cocoyer et al. 2006). Other suggested applications are found into optical storage (Oliveira et al. 2005), self-organized memories (Barille et al. 2006), and self-healing structures (Mazaheri et al. 2016).

Unlike the initial belief that azo-dyes should be covalently attached to polymers in order to perform efficiently like stable photo-activated materials, it has been shown that they could be hydrogen- or halogen-bonded to a polymer host (Priimagi et al. 2012). It has also been shown that to function efficiently the only requirement is that the material forms a glass (Kirby et al. 2014; Shirota 2000), which could also be justified by molecular dynamics simulation (Teboul et al. 2009).

Our aim in this paper is to develop new assemblies based on natural polymers bond to azo-dyes in order to build on the availability of natural products. The azo-dye Disperse 1 (DR1; Fig. 1a) could be bond to DNA by using the surfactant cetyltrimethylammonium chloride (CTMA) as a compatibility agent (Czaplicki et al. 2007). In this case, CTMA is attached ionically on both sides of the DNA stands, so both DNA-CTMA complex and dye can be dissolved in organic solvent. In this work, we propose a new scheme for DR1 - cellulose derivative matrix bonding into which no compatibility agent is necessary. Cellulose is one of the most abundant polymers in our environment, but its main disadvantage is insolubility property. Therefore, to be useful, many different water- 
soluble derivatives were developed. Among them hydroxypropyl cellulose (HPC; Fig. 1b) is unique and a very interesting one because it is soluble not only in water but also in some organic solvents like acetone (Assis et al. 2015; Rinaudo 2016). Moreover, it is a natural polymer derivative, so it is widely employed in the food (Sebti et al. 2002), pharmaceutical (Tanaka et al. 2017; Wei et al. 2017) and cosmetics industries (Prado and Matulewicz 2014) like thickening or encapsulating agent. On the other hand, it has also been investigated as polymer electrolyte matrix for all solid electrochromic windows (Assis et al. 2015; Ledwon et al. 2015).

The objective of our research is to demonstrate that dissolution of an azo-dye in a solid matrix can be promoted by light. Technically, both DR1 and HPC well dissolve in acetone, but after mixing and drying processes, DR1 forms aggregates (light and dark regions) in HPC matrix, which is seen in Fig. 2. However, when this deposit is illuminated with visible-light a solubilization of DR1 in HPC matrix is observed, and this process is reversible. This shows that DR1 can be incorporated into HPC using a reversible photoinduced dispersion process, which demonstrates our hypothesis. Furthermore, the process reveals new features of the light induced repeated isomery process of DR1 in HPC matrix, which can be used for photo-activated melting and dispersion of dyes in natural macromolecules-based materials. The process is likely to be extended to other natural polymers and composites (Zerria et al., 2016).

\section{Experimental}

The hydroxyethyl cellulose (HPC; CAS number 9004-64-2) was purchased from SigmaAldrich under catalogue number 435007. This macromolecule was received as a powder with $99 \%$ of 20 mesh particle size, had a weight average molecular weight $\left(\mathrm{M}_{\mathrm{w}}\right)$ of 80,000 , number average molecular weight $\left(\mathrm{M}_{\mathrm{n}}\right)$ of 10,000 , less than $5 \mathrm{wt} . \%$ of impurities, and viscosity of $250-800 \mathrm{cps}$ when dissolved at $10 \mathrm{wt} \%$ in water at $25{ }^{\circ} \mathrm{C}$ (SigmaAldrich-Merck 2017). HPC-based samples were prepared by dissolving $0.3 \mathrm{~g}$ of HPC in $25 \mathrm{~mL}$ of acetone (Aldrich) under a magnetic stirrer overnight. Then, 2, 6, or $8 \mathrm{mg}$ of Disperse Red 1 (DR 1; Aldrich) was dissolved in $5 \mathrm{~mL}$ of acetone for few minutes, added to HPC solution, and stirred for few more minutes until complete dissolution. Then, a homogenous red color solution was poured on glass Petri dish and cast at room temperature overnight to form a uniform polymer-dye deposition as shown in Fig. 2. 
HPC sample shows a $16 \%$ weight loss up to $150{ }^{\circ} \mathrm{C}$, which is attributed to the loss of absorbed water (Fig. S1). The subsequent loss of $71 \%$ of weight in the temperature range of 265 to $445{ }^{\circ} \mathrm{C}$ (DTG peak at $386{ }^{\circ} \mathrm{C}$ ) was attributed to the thermal degradation of the polymer (Cavallaro et al. 2011). A similar behavior is observed for the sample of HPC-DR1, but the absorbed water release ends up at $130{ }^{\circ} \mathrm{C}$. During degradation, the sample loses $61 \%$ mass. At $1000{ }^{\circ} \mathrm{C}$ the remaining ashes are 1 and $16 \%$ of the initial masses of HPC and HPC-DR1 samples, respectively.

The samples were illuminated over a $2 \mathrm{~cm}$-diameter spot using a green LED (Light Emission Diode) reflector Briwax FCG-50W ( $\lambda=525 \mathrm{~nm}$; spectrum of this lamp is shown in Fig. S2) with light intensity over the sample of $6.6 \mathrm{~W}$. These experiments were also performed with laser Luzchem Spectroradiometer SPR-01-235-850 nm at $514.5 \mathrm{~nm}$ on a $0.6 \mathrm{~cm}$-diameter spot, with light intensity over the sample of $1.6 \mathrm{~W}$. Illumination time varied from $10 \mathrm{~s}$ to $4 \mathrm{~min}$.

UV-Vis spectra were collected with Jasco V770 spectrophotometer that operated in absorbance mode between 300 and $800 \mathrm{~nm}$ wavelength range. The LED or laser illuminated samples spots on Petri dish were placed close to the detector to avoid light scattering.

\section{Results and Discussion}

Fig. 3 shows pictures of illumination spots for increased times on two different samples: HPC-DR $18 \mathrm{mg}$ illuminated under LED (Fig. 3a) and HPC-DR1 $2 \mathrm{mg}$ under laser light (Fig. 3b). In both cases, the samples get darker under increased illumination time. The effect is permanent and almost stable forever if nothing else happens to the sample. We could keep darkened samples for several months, without seeing any change. The effect could be attributed to heating under light. The temperature under prolonged LED illumination indeed reaches up to $80{ }^{\circ} \mathrm{C}$ after prolonged illumination, and we verified that the samples also darken upon prolonged heating to temperatures above $60{ }^{\circ} \mathrm{C}$ when placed on heating plate. One can suggest that this could be related to the glass transition ( $\mathrm{Tg})$ of HPC, but it is not happening because HPC Tg is $205^{\circ} \mathrm{C}$. Furthermore, to exclude heating impact on observed phenomena we subjected the samples to $525 \mathrm{~nm}$ laser beam (Fig. S3). Additionally, we monitored the temperature under the laser beam, and we stated that it does not increase significantly. Therefore, we can assume there is no influence of temperature when the sample is exposed to $514.5 \mathrm{~nm}$ laser beam (Fig 3b). However, the 
darkening effect is clearly visible. So, there is evidence of a light induced darkening effect. The darkening effect can be reverted by wetting the sample, which can again be darkened under light.

Fig. 4 shows the UV-vis spectra of the HPC-DR1 2 mg sample shown in Fig. $3 \mathrm{~b}$ for different illumination times under laser light. The cut-off at $300 \mathrm{~nm}$ is the glass substrate. HPC does not show any significant absorption in the visible (Fig. S4). We clearly see two features coming from the DR1 molecule: one at $500 \mathrm{~nm}$ is the same as the absorption from DR1 in solution (Delysse et al. 1997). The second one at $400 \mathrm{~nm}$ was previously attributed to DR1 aggregation (Choe et al. 2000; Marino et al. 2008). The observation is that upon illumination the peak at $400 \mathrm{~nm}$ disappears and the peak at $500 \mathrm{~nm}$ increases. The increase of the absorption at $500 \mathrm{~nm}$ totally justifies the darkening observed in Fig. $3 \mathrm{~b}$, in which the transmission of the illuminated spots was reduced by up to $80 \%$. This suggests that light breaks the DR1-aggregates formed into the HPS host during drying process. The aggregates have low absorption in the green light range owing to the large permanent dipole of DR1 which forces H-type aggregation. When the aggregates are dissolved, the intense red color of DR1 dye is restored.

In order to sustain the claim, we monitored the Fourier Transform Infrared (FTIR) spectrum of HPC, HPC-DR1 2mg as cast and HPC-DR1 $2 \mathrm{mg}$ heated at $60{ }^{\circ} \mathrm{C}$ (Fig. 5). Literature (Cinar et al. 2011) teaches us that aromatic nitro compounds have strong absorptions owing to the asymmetric and symmetric stretching vibrations of the $\mathrm{NO}_{2}$ group at $1485-1570$ and $1320-1370 \mathrm{~cm}^{-1}$, respectively. We see in Fig.5 new and strong bands that appeared at 1600,1508 , and $1342 \mathrm{~cm}^{-1}$ after darkening the sample by heating at $60{ }^{\circ} \mathrm{C}$. They are assigned to symmetric and asymmetric stretching modes of $\mathrm{NO}_{2}$, which were not visible in the aggregated form owing to the higher symmetry of the aggregates. There also is a new band at $3274 \mathrm{~cm}^{-1}$, which is attributed to the amorphous DR1. This band is also expected because of loss of sample order upon heating. This confirms the attribution of sample darkening in Fig. 3 to the breaking and dissolution of DR1aggregates into the HPC matrix.

We also studied the Raman spectrum of HPC-2 and 8 mg DR1 samples (Fig. S5). We did not observe any noticeable changes upon darkening. The spectral features are the same as observed in previously published results (Lagugné Labarthet and Sourisseau 1996).

The reversibility of the process was investigated by UV-Vis spectroscopy. We see in Fig. 6 that after wetting the sample the strong absorbance bleaches by almost half of 
its value. Then, upon further illumination absorbance is gradually restored. Technically, absorbance was not completely restored to its initial values in that particular trial owing to some degradation of the film quality upon wetting; we noticed that a meniscus formed around the wetted area.

Fig. 7 illustrates the mechanism proposed to interpret reversible light-induced solubility of DR1 dye in a HPC matrix. The experiment shows that DR1 is not spontaneously soluble at room temperature in HPC; it forms aggregates, as illustrated in the left part of Fig.7. Aggregates are dissolved under illumination with visible light. This is promoted by repeated trans-cis isomery, which induces photo-fluidity inside the polymer matrix, breaking the cage in which aggregated molecules are trapped (Teboul et al. 2011). We assume that once dissolved, the molecules get stabilized by hydrogen bonding of the DR1-hydroxyl group to the available ether-groups of HPC. This explains that the darkened form in the right part of Fig.7 be indefinitely stable in the dark. We also understand that the bonding does not take place spontaneously owing to the intra molecular hydrogen bonding that happens naturally in HPC and saturates the available ethers. This explains that DR1 is not spontaneously soluble at room temperature in HPC. Water swells HPC, restoring H-bonds to their initial state after drying, which explains reversibility of the process.

\section{Conclusions}

In a present study, we have prepared coatings of Disperse Red 1 dye in a hydroxypropyl cellulose matrix, which is a natural polymer derivative. We have observed that beside both molecules are soluble in organic solvent, after its evaporation Disperse Red 1 forms aggregates in polymer matrix. To improve the compatibility between molecules we have illuminated the samples with visible light, and we have observed a dissolution of the dye. This new phenomenon involving organic dye and natural polymer derivative was confirmed by visible light and FTIR spectroscopies. UV-Vis spectra confirmed disappearance of aggregates blue-shifted band at $400 \mathrm{~nm}$ after illumination of the samples with visible light and appearance of FTIR bands at 1600,1508 , and $1342 \mathrm{~cm}^{-1}$ assigned to symmetric and asymmetric stretching modes of $\mathrm{NO}_{2}$ of not aggregated DR1. Therefore, we have evidenced the process by which solubilization of a dye in a solid matrix was triggered by the action of light. This happens owing to repeated trans-cis isomery. The process is a new feature of the light induced molecular movements of azo-dyes. It may 
be used practically for the photo-command of polymer properties and/or light storage.

The process is likely to be extended to other natural polymers and composites, which may be amenable to photonic applications. It also reveals the strong potential of azo-materials as light powered molecular motors.

\section{Acknowledgments}

The authors are grateful to FAPESP (grants no. 2016/13184-0 and 2014/17174-4) for financial support given for this research work and to Marcos Cardoso and Prof. Cleber Mendonça, from IFSC-Universidade de São Paulo, for laser lab facility. A. Pawlicka also thanks CNPq for research grant Produtividade em Pesquisa no. 305029/2013-4.

\section{References}

Apostoluk A, Chapron D, Gadret G, Sahraoui B, Nunzi J-M, Fiorini-Debuisschert C, Raimond P (2002) Quasi-phase-matched gratings printed by all-optical poling in polymer films Opt Lett 27:2028-2030 doi:https://doi.org/10.1364/OL.27.002028

Assis LMN, Sabadini RC, Santos LP, Kanicki J, Łapkowski M, Pawlicka A (2015) Electrochromic device with Prussian blue and HPC-based electrolyte Electrochim Acta 182:878-883 doi:https://doi.org/10.1016/j.electacta.2015.09.133

Barille R, Ahmadi-Kandjani S, Ortyl E, Kucharski S, Nunzi J-M (2006) Cognitive ability experiment with photosensitive organic molecular thin films Phys Rev Lett 97:048701 doi:https://doi.org/10.1103/PhysRevLett.97.048701

Cavallaro G, Donato DI, Lazzara G, Milioto S (2011) Films of halloysite nanotubes sandwiched between two layers of biopolymer: from the morphology to the dielectric, thermal, transparency, and wettability properties J Phys Chem C 115:20491-20498 doi:https://doi.org/10.1021/jp207261r

Choe DH, Jo GJ, Cha YG (2000) Optically induced anisotropy in photoresponsive solgel matrix bearing a silylated disperse red 1 B Korean Chem Soc 21:1222-1226

Cinar M, Coruh A, Karabacak M (2011) FT-IR, UV-vis, 1 H and 13 C NMR spectra and the equilibrium structure of organic dye molecule disperse red 1 acrylate: a combined experimental and theoretical analysis Spectrochimica Acta Part A: Molecular and Biomolecular Spectroscopy 83:561-569 doi:https://doi.org/10.1016/j.saa.2011.09.003

Cocoyer $\mathrm{C}$ et al. (2006) Implementation of submicrometric periodic surface structures toward improvement of organic-solar-cell performances Appl Phys Lett 88:133108 doi:http://dx.doi.org/10.1063/1.2188600

Cojocariu C, Rochon P (2004) Light-induced motions in azobenzene-containing polymers Pure Appl Chem 76:1479-1497 doi:https://doi.org/10.1351/pac200476071479

Czaplicki R, Krupka O, Essaidi Z, El-Ghayoury A, Kajzar F, Grote J, Sahraoui B (2007) Grating inscription in picosecond regime in thin films of functionalized DNA Opt Express 15:15268-15273 doi:https://doi.org/10.1364/OE.15.015268 
Delysse S, Raimond P, Nunzi J-M (1997) Two-photon absorption in noncentrosymmetric dyes Chem Phys 219:341-351 doi:https://doi.org/10.1016/S0301-0104(97)00106-7

Hubert C, Fiorini-Debuisschert C, Rocha L, Raimond P, Nunzi J-M Emission properties of organic light-emitting diodes directly patterned using optically controlled nanostructuration means. In: Photonics Europe, 2004. International Society for Optics and Photonics, pp 441-448. doi:https://doi.org/10.1117/12.552118

Kirby R, Sabat RG, Nunzi J-M, Lebel O (2014) Disperse and disordered: a mexylaminotriazine-substituted azobenzene derivative with superior glass and surface relief grating formation J Mater Chem C 2:841-847 doi:https://doi.org/10.1039/C3TC32034K

Lagugné Labarthet F, Sourisseau C (1996) Raman Study of the Photoisomerization and Angular Reorientation of Azobenzene Molecules in a DR1- Doped Polymer Matrix Journal of Raman spectroscopy 27:491-498 doi:https://doi.org/10.1002/(SICI)1097-4555(199606)27:6<491::AIDJRS988>3.0.CO;2-G

Ledwon P, Andrade JR, Lapkowski M, Pawlicka A (2015) Hydroxypropyl cellulosebased gel electrolyte for electrochromic devices Electrochim Acta 159:227-233 doi:https://doi.org/10.1016/j.electacta.2015.01.168

Marino I-G, Raschellà R, Lottici PP, Bersani D (2008) Chromophore aggregation and photoinduced dichroism in sol-gel films J Non-Cryst Solids 354:688-692 doi:https://doi.org/10.1016/i.jnoncrysol.2007.07.072

Mazaheri L, Sabat RG, Lebel O, Nunzi J-M (2016) Unraveling the nucleation and growth of spontaneous surface relief gratings Optical Mat 62:378-391 doi:https://doi.org/10.1016/j.optmat.2016.10.003

Oliveira ON, dos Santos DS, Balogh DT, Zucolotto V, Mendonça CR (2005) Optical storage and surface-relief gratings in azobenzene-containing nanostructured films Advances in colloid and interface science 116:179-192 doi:https://doi.org/10.1016/j.cis.2005.05.008

Prado HJ, Matulewicz MC (2014) Cationization of polysaccharides: A path to greener derivatives with many industrial applications Europ Polym J 52:53-75 doi:https://doi.org/10.1016/j.eurpolymj.2013.12.011

Priimagi A et al. (2012) Halogen Bonding versus Hydrogen Bonding in Driving SelfAssembly and Performance of Light- Responsive Supramolecular Polymers Adv Funct Mater 22:2572-2579 doi:https://doi.org/10.1002/adfm.201200135

Rinaudo M (2016) Physicochemical behaviour of semi-rigid biopolymers in aqueous medium Food Hydrocoll doi:https://doi.org/10.1016/j.foodhyd.2016.09.015

Sebti I, Ham-Pichavant F, Coma V (2002) Edible bioactive fatty acid-cellulosic derivative composites used in food-packaging applications J Agricult Food Chem 50:4290-4294 doi:https://doi.org/10.1021/jf0115488

Sentein C, Fiorini C, Lorin A, Nunzi JM (1997) Molecular rectification in oriented polymer structures Adv Mater 9:809-811 doi:https://doi.org/10.1002/adma.19970091009

Shirota Y (2000) Organic materials for electronic and optoelectronic devices Basis of a presentation given at Materials Chemistry Discussion No. 2, 13-15 September 1999, University of Nottingham, UK J Mater Chem 10:1-25 doi:https://doi.org/10.1039/A908130E

Sigma-Aldrich-Merck (2017) Hydroxypropyl cellulose. Sigma-Alfrich-Merck. http://www.sigmaaldrich.com/catalog/product/aldrich/435007?lang=en\&region=

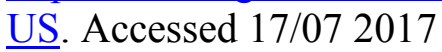


Tanaka A et al. (2017) Nasal drug absorption from powder formulations: The effect of three types of hydroxypropyl cellulose (HPC) Europ J Pharmaceut Sci 96:284289 doi:https://doi.org/10.1016/j.ejps.2016.09.028

Teboul V, Saiddine M, Nunzi J-M (2009) Isomerization-induced dynamic heterogeneity in a glass former below and above T g Phys Rev Lett 103:265701 doi:https://doi.org/10.1103/PhysRevLett.103.265701

Teboul V, Saiddine M, Nunzi J-M, Accary J-B (2011) An isomerization-induced cagebreaking process in a molecular glass former below Tg The Journal of Chemical Physics 134:114517 doi:http://dx.doi.org/10.1063/1.3563548

Viswanathan N, Kim D, Tripathy S (1999) Surface relief structures on azo polymer films J Mater Chem 9:1941-1955 doi:https://doi.org/10.1039/A902424G

Wei S, Ma Y, Luo J, He X, Yue P, Guan Z, Yang M (2017) Hydroxypropylcellulose as matrix carrier for novel cage-like microparticles prepared by spray-freeze-drying technology Carbohydrate Polym 157:953-961 doi:https://doi.org/10.1016/j.carbpol.2016.10.043

Zilker SJ, Bieringer T, Haarer D, Stein RS, van Egmond JW, Kostromine SG (1998) Holographic data storage in amorphous polymers Adv Mater 10:855-859 doi:https://doi.org/10.1002/(SICI)1521-4095(199808)10:11<855::AIDADMA855>3.0.CO;2-H 
Figures Captions

Fig. 1. Chemical formulas of DR1 (a) and hydroxypropyl cellulose (b).

Fig. 2. Picture of HPC-DR1 (2 mg) sample on glass Petri dish (a) and picture showing aggregates from sample with $8 \mathrm{mg}$ of DR1 (b).

Fig. 3. Picture of HPC-DR1 (8 mg) illuminated with green LED lamp (a) HPC-DR1 (2 $\mathrm{mg}$ ) illuminated with green laser beam (b).

Fig. 4. UV-Vis spectra of HPC-DR1 $2 \mathrm{mg}$ sample, as prepared and illuminated under laser for 5, 10, 15, and $20 \mathrm{~s}$.

Fig. 5. FTIR spectrum of HPC, HPC-DR1 2mg, and HPC-DR1 $2 \mathrm{mg}$ heated above $60{ }^{\circ} \mathrm{C}$ till darkening.

Fig. 6. UV-Vis spectra of HPC-DR1 $2 \mathrm{mg}$ sample after prolonged LED illumination (A; red), after wetting and subsequent drying (D; green), followed by 1 min LED illumination (C; yellow) and 3 min LED illumination (B; blue).

Fig. 7. Representation of reversible light-induced solubility of Disperse Red 1 dye in hydroxypropylcellulose matrix. Continues lines represent HPC chains and red dots dissolved DR1 molecules.

\section{Supplementary Material}

Fig. S1. LED lamp spectral characteristics.

Fig. S2. Setup of illumination of the sample with laser beam experiment (a) and sample on the support (b).

Fig. S3. UV-Vis spectra of HPC (black line) and HPC-DR1 (red line) in acetone solutions. 
Fig. S4. Raman spectra of DR1 (red), HPC-DR1 2mg (blue) and HPC-DR1 8mg heated at $60^{\circ} \mathrm{C}$ (green). 
(b)

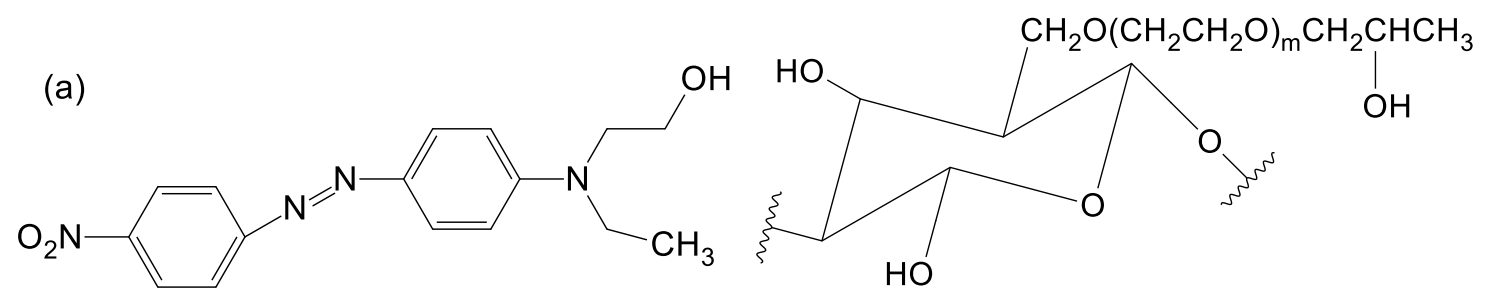


Figure $2 b$

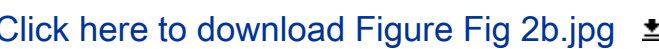
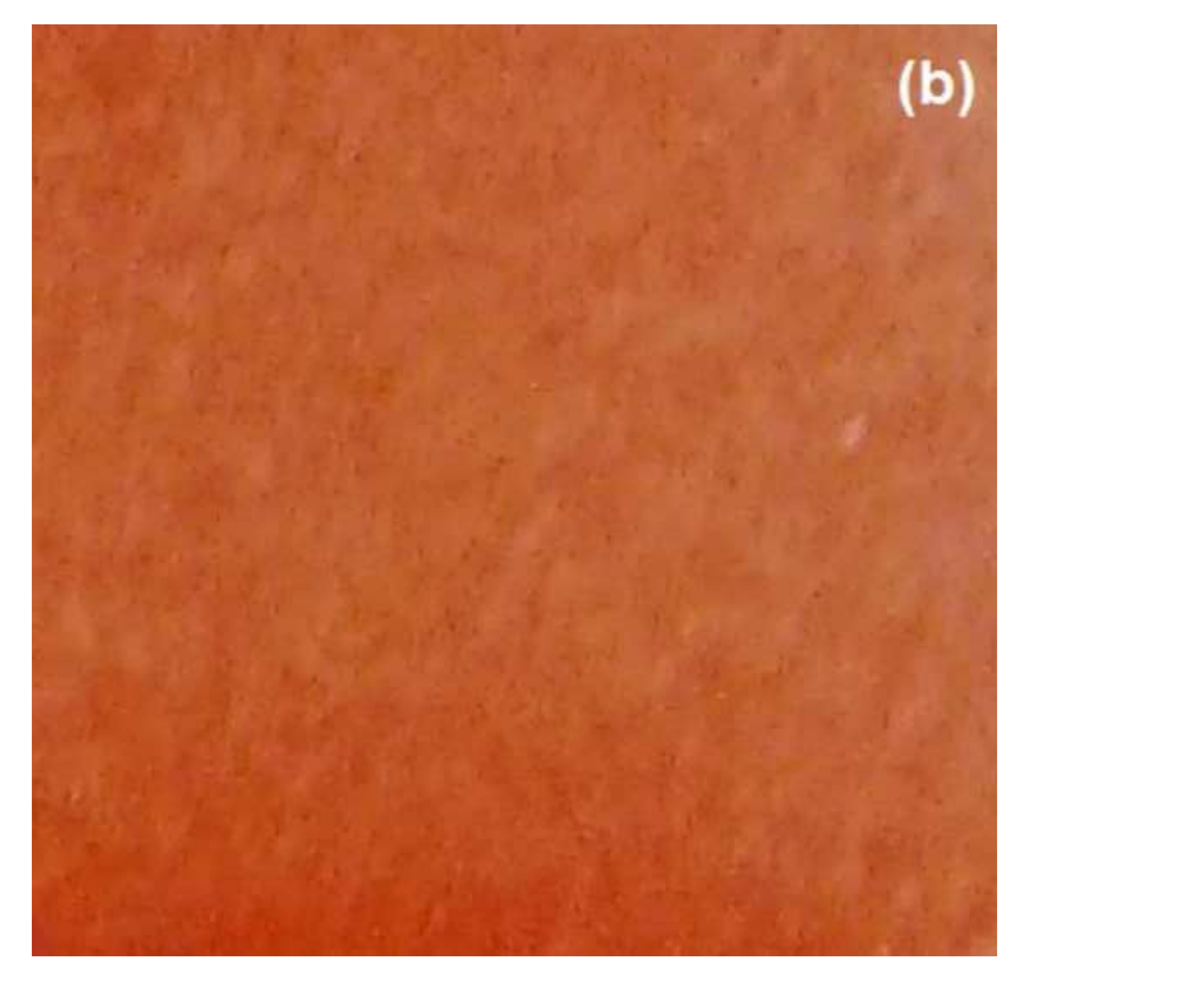 \\ (b)}

$-1$

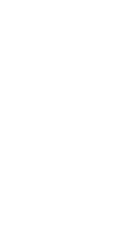


Click here to download Figure Fig 3a.jpg $\underline{\underline{\underline{ }}}$

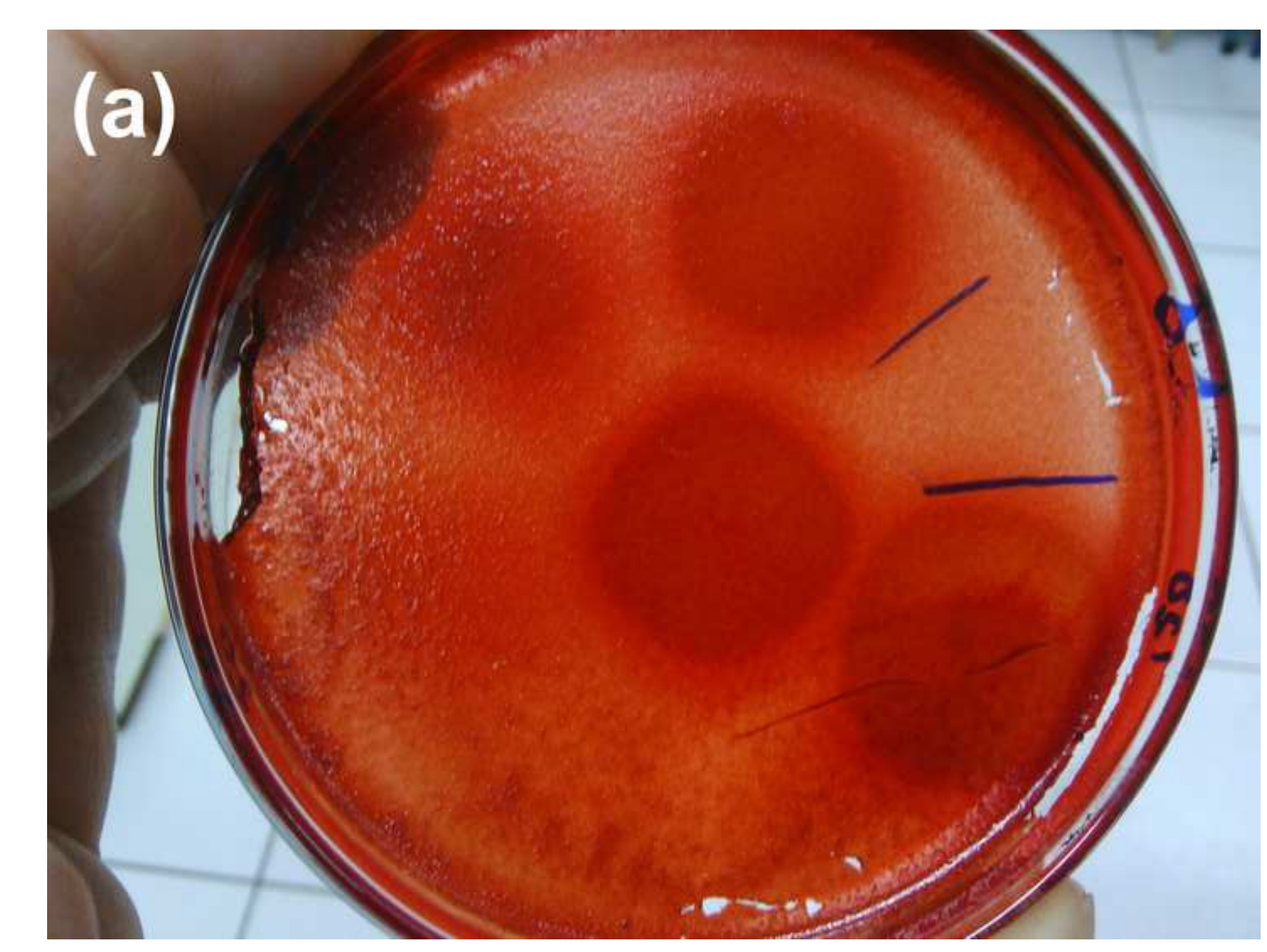


Figure $3 b$

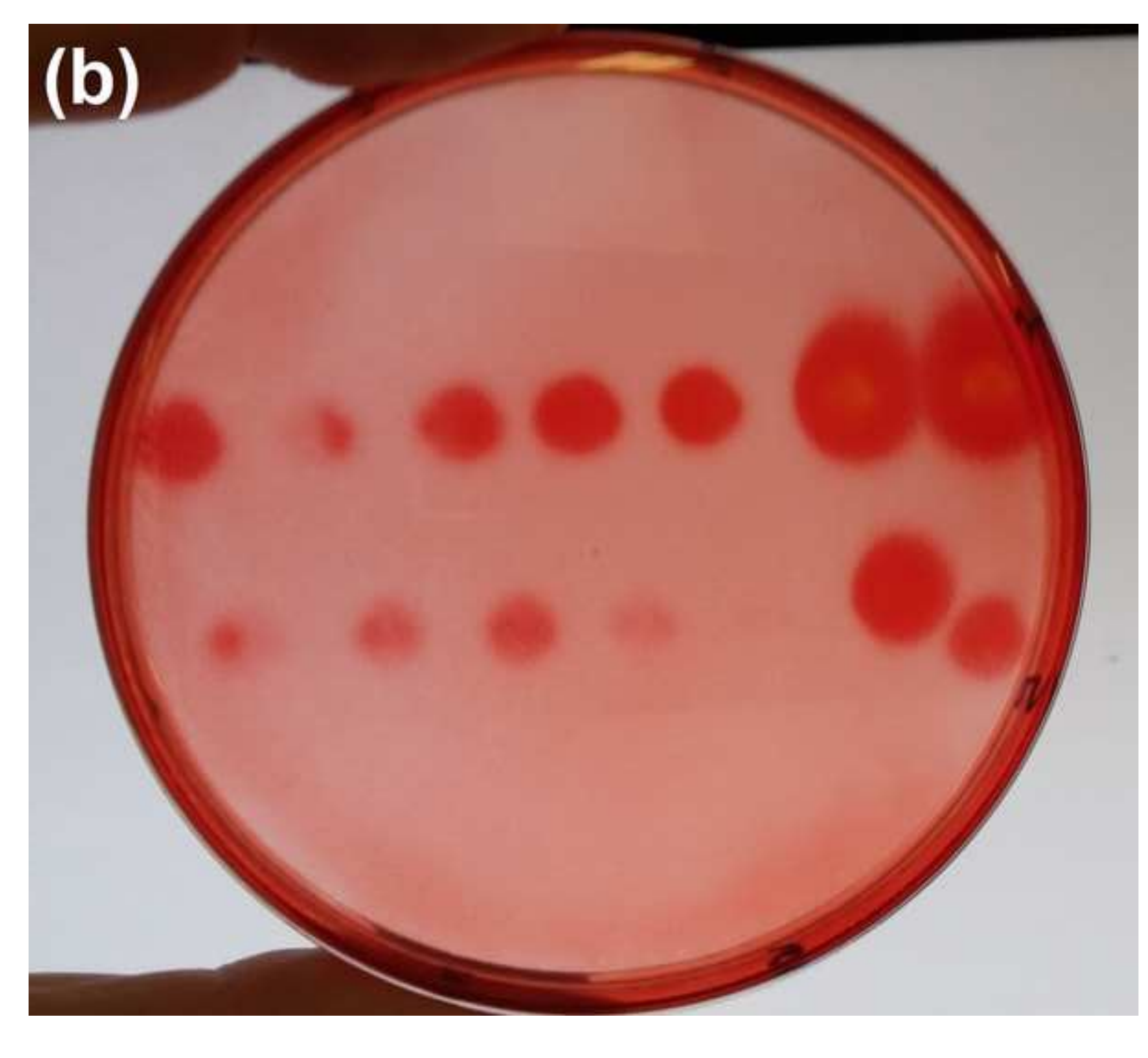

Click here to download Figure Fig 3b.jpg $\stackrel{\star}{\underline{*}}$

(⿸丆口
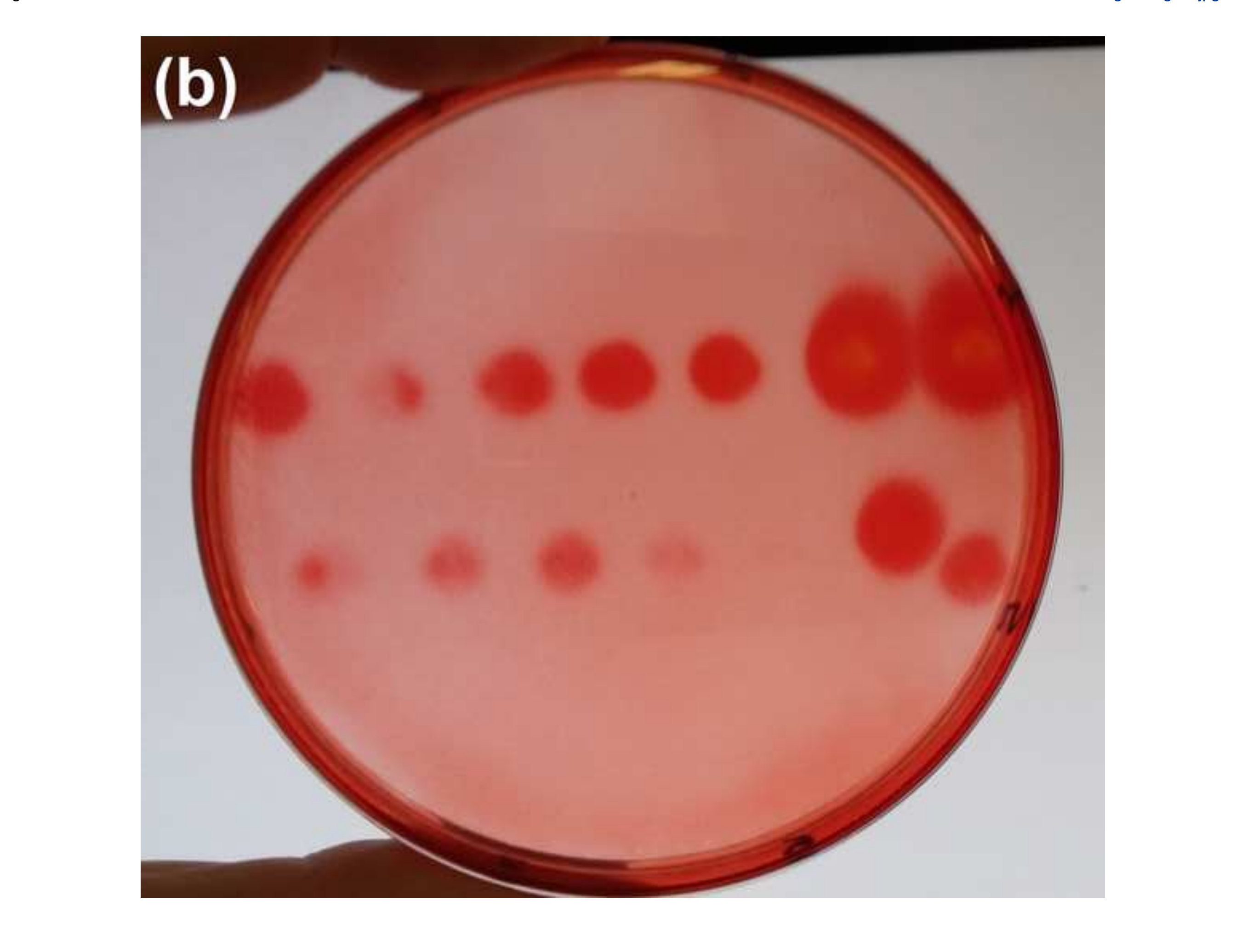


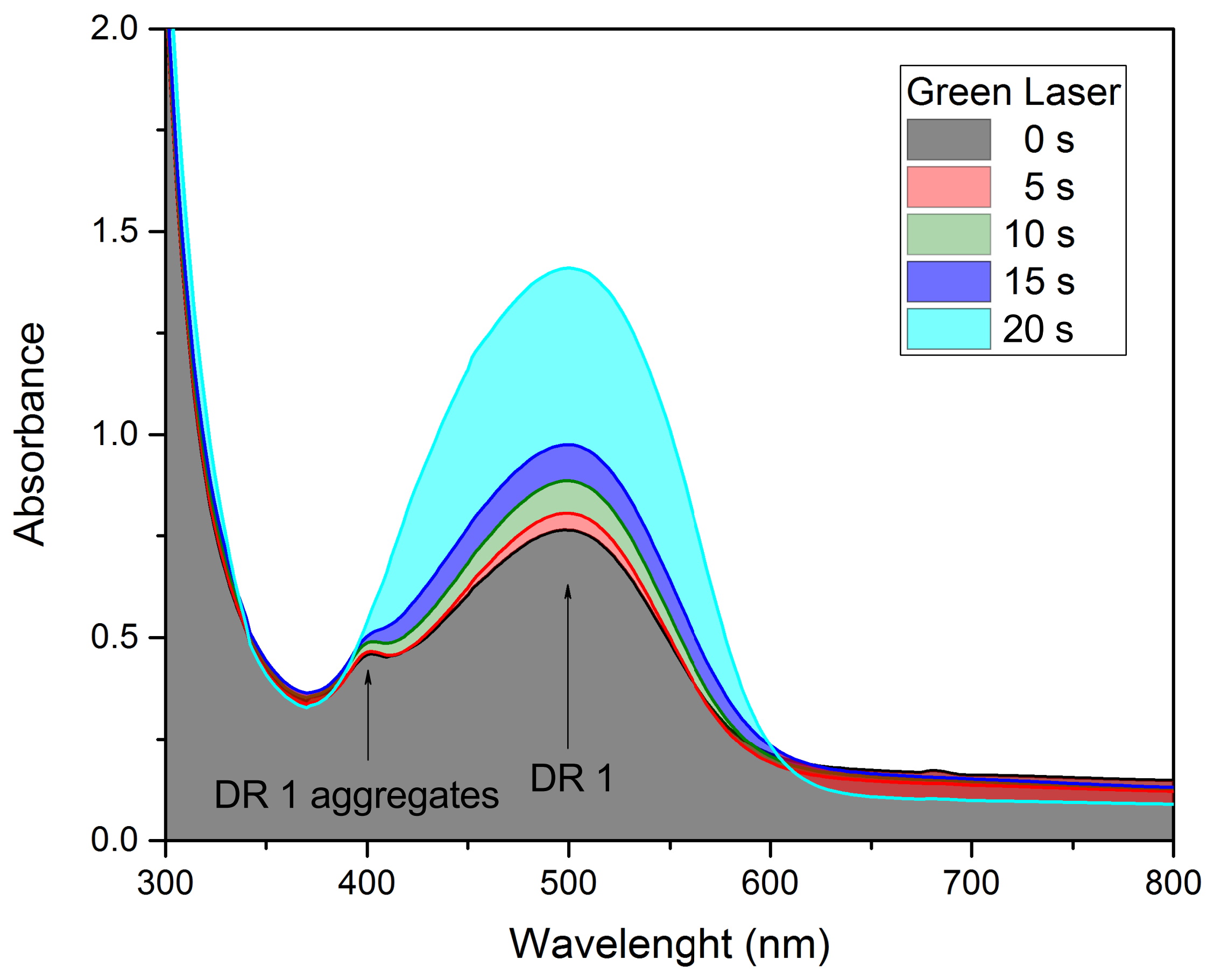




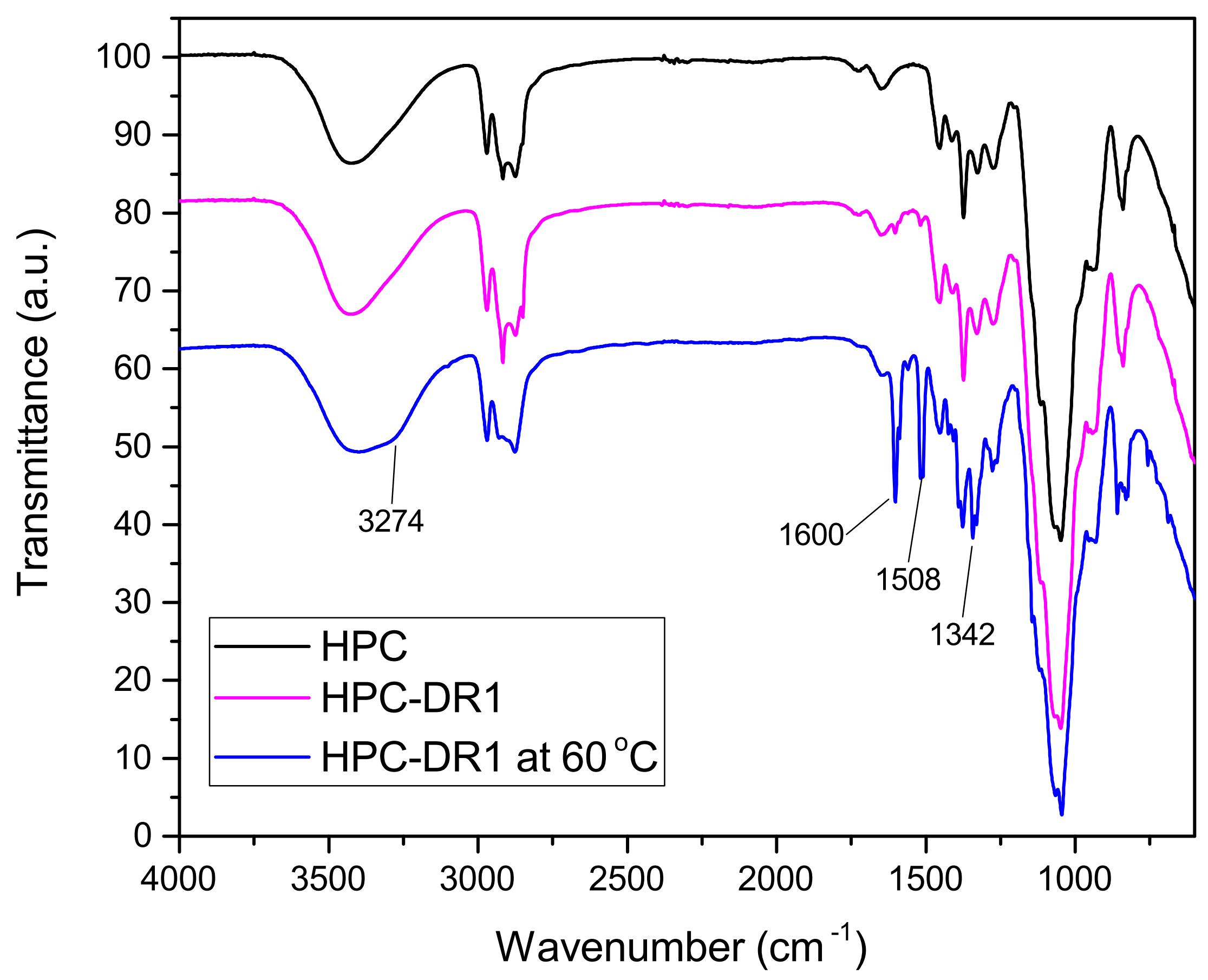




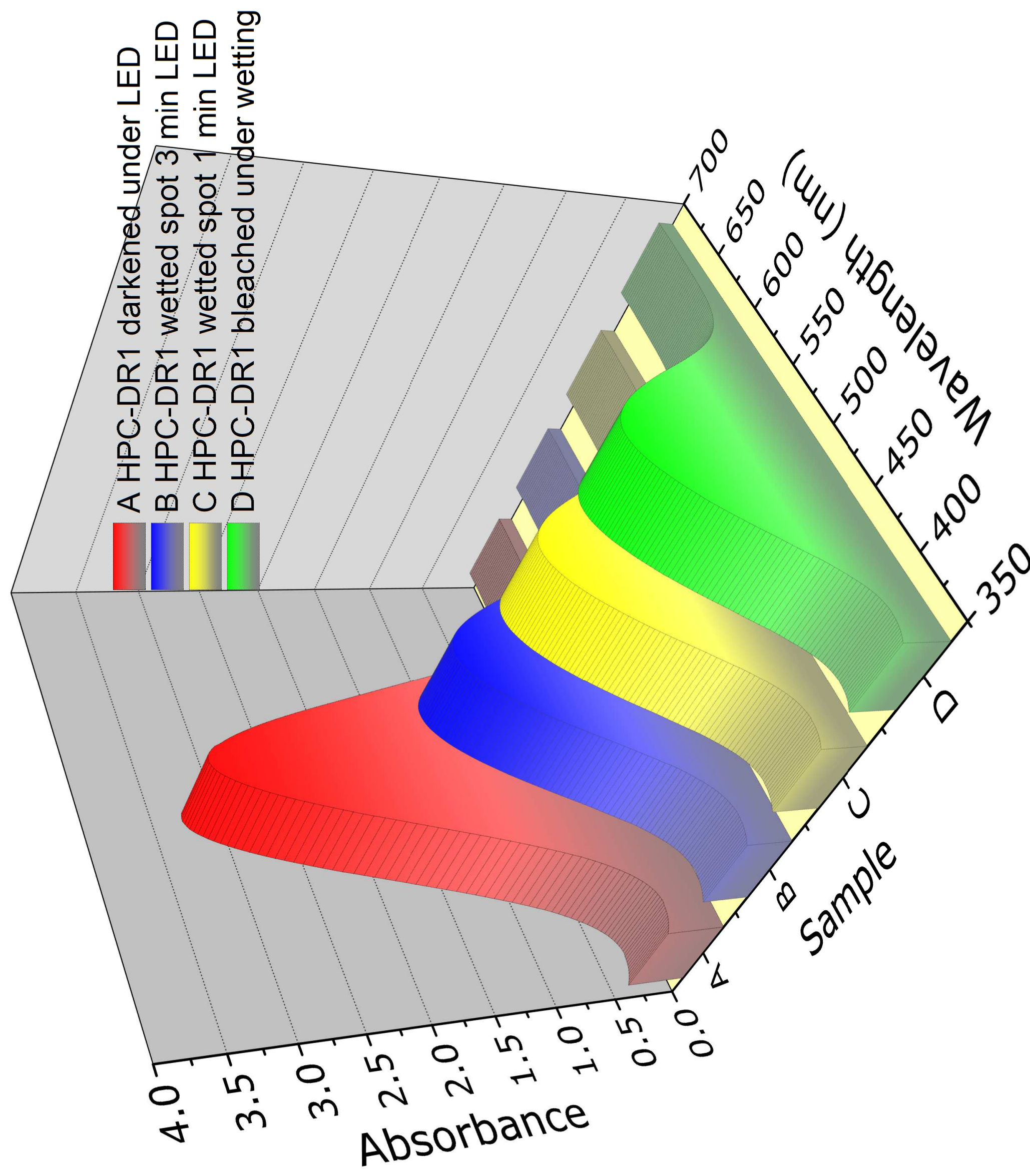

